\title{
Short-term high-temperature creep of Grade 5 titanium alloy heated in air and argon
}

\author{
L.M. Zamaraev \\ leva.zam@mail.ru \\ Institute of Engineering Science, UB RAS, 34 Komsomolskaya St., Ekaterinburg, 620049, Russia
}

\begin{abstract}
The paper presents the results of studying the deformation of samples of the alloy Grade 5 while they are heated to temperatures ranging between 900 and $1350 \mathrm{~K}$ with constant tensile stresses of 4.45, 6.91 and 9.36 $\mathrm{MPa}$, which is significantly below the yield stress of the alloy at the test temperatures $\left(\sigma_{\mathrm{T}} \geq 34 \mathrm{MPa}\right)$. Under these conditions, the deformation occurs due to the mechanism of short-term creep. During testing, the samples are heated in air and argon environments. The results are compared with the results obtained earlier in similar studies for Grade 2 commercially pure titanium and Ti-5AL. The investigation has revealed a qualitative similarity of the effect of environment on the processes of creep deformation in Grade 2 commercially pure titanium, Ti-5AL alloy, and Grade 5 alloy. Herewith, all the alloys under study have close values of activation energy (from 250 to $320 \mathrm{~kJ} / \mathrm{mol}$ ). The experiments have shown that, as compared to previously studied alloys, Grade 5 alloy has higher temperatures of the onset of creep deformation and lower creep rates. Processing of the results has allowed us to evaluate the empirical coefficients for Grade 5 alloy in two dependences obtained earlier for the Ti-5AL and Grade 2 alloys. One dependence relates the creep rate to the heating temperature, tensile stress, and creep activation energy; the other one relates the creep deformation onset temperature (residual creep deformation within 1 hour of holding is $0.2 \%$ ) to the conventional short-term creep limit.
\end{abstract}

Keywords: short-term high-temperature creep, conventional creep limit, creep rate.

УДК: 669.018

\section{Кратковременная высокотемпературная ползучесть титанового сплава Grade 5 при нагреве в воздухе и аргоне}

\author{
Замараев Л.М. \\ Институт машиноведения, УрО РАН, ул. Комсомольская, 34, Екатеринбург, 620049, Россия
}

В статье представлены результаты исследований деформации образцов из сплава Grade 5 в процессе их нагрева до температур 900-1350 К при постоянных растягивающих напряжениях 4.45, 6.91 и 9.36 МПа, что значительно ниже предела текучести сплава при температурах исследований ( $\sigma_{\mathrm{T}} \geq 34 \mathrm{MПа).} \mathrm{При} \mathrm{таких} \mathrm{условиях} \mathrm{деформация}$ будет осуществляться за счет механизма кратковременной ползучести. В процессе исследований нагрев образцов проводился в воздушной и аргоновой средах. Полученные результаты сравнивались с ранее проведенными аналогичными исследованиями для технически чистого титана Grade 2 и Ti-5AL. Проведенные исследования выявили качественную аналогию влияния среды на процессы деформации ползучести для технически чистого титана Grade 2, сплава Ti-5AL и сплава Grade 5. При этом все исследованные сплавы имели близкие значения энергии активации (250-320 кДж/моль). Эксперименты показали, что сплав Grade 5, по сравнению с ранее исследованными сплавами, имеет более высокие температуры начала деформации ползучести и низкие скорости ползучести. Обработка полученных результатов позволила определить для сплава Grade 5 значения эмпирических коэффициентов в двух ранее полученных для сплавов Ti-5AL и Grade 2 зависимостях. В зависимости, связывающей скорость ползучести с температурой нагрева, напряжением растяжения и энергией активации ползучести, а также в зависимости температуры начала деформации ползучести (остаточная деформация ползучести в течение 1 часа выдержки составляет $0.2 \%$ ) от значения условного предела кратковременной ползучести.

Ключевые слова: кратковременная высокотемпературная ползучесть, условный предел ползучести, скорость ползучести. 


\section{1. Введение}

Процессы ползучести являются нежелательным фактором при эксплуатации конструкций и механизмов и могут существенно ограничить срок их службы.

С другой стороны, при металлообработке в ряде случаев ползучесть может быть использована для осуществления формообразующих операций, например, при штамповке деталей сложной геометрии в условиях кратковременной высокотемпературной ползучести $[1,2]$.

Важность этих процессов обусловила большой интерес к их изучению. Ряд публикаций описывают результаты исследований титановых сплавов при ползучести в широком диапазоне температур, начиная с комнатных [3] до высоких, при статическом и циклическом характере нагружений [4].

В исследованиях определены общие аналитические уравнения в виде экспоненциальных, степенных и логарифмических зависимостей, описывающих связь скорости (степени) деформации ползучести при постоянных значениях температуры и величины приложенного напряжения [5-6].

Для титана и его сплавов характерно наличие активного газопоглощения водорода, азота и кислорода. Поэтому большинство исследований посвящено ползучести титановых сплавов в перечисленных выше активных средах. Эти газы, как правило, снижают скорость ползучести за счет образования с титаном твердых растворов внедрения и высокопрочных частиц оксидов, гидридов и нитридов, которые после охлаждения могут существенным образом изменять физико-механические свойства сплавов [7-15].

Сведения о влиянии нейтральных газовых сред на ползучесть титана и его сплавов в научно-технических публикациях крайне немногочисленны.

Из известных следует отметить исследования авторов работ [16,17], которые установили, что долговечность при ползучести $(\alpha+\beta)$ сплава Ti-6Al-4V в аргоне выше, чем в воздухе из-за отсутствия окисления поверхности образцов. Снижение циклической долговечности в вакууме по сравнению с воздухом, обнаруженное в [19], также объяснялось ускорением накопления поврежденности в окислительной среде.

Целью данной статьи являлось изучение закономерностей кратковременной высокотемпературной ползучести сплава Grade 5 в аргоновой и воздушной средах.

\section{2. Материалы}

Образцы для исследований были изготовлены из горячепрессованных прутков диаметром 12 мм сплава Grade 5 (ВТ6 по российскому ГОСТу). Химический состав сплава: $\mathrm{Al}-5.563 \%, \mathrm{Si}-0.095 \%$, Fe - 0.5\%, $\mathrm{C}-0.09 \%, \mathrm{O}_{2}-0.18 \%, \mathrm{H}_{2}-0.012 \%, \mathrm{~V}-4.23 \%$, $\mathrm{Zr}-0.13 \%, \mathrm{Ti}$ - остальное.

Образцы для испытаний на ползучесть имели размеры рабочей части: длина 50 мм, диаметр 5 мм. Для крепления образцов в захватах испытательной установки они имели на концах утолщения диаметром 8 мм с нарезанной резьбой. До тестирования все образцы были отожжены в вакуумной печи при температуре 900 К в течение 1 часа. Эксперименты проводились в воздушной и аргоновой средах. Для проведения испытаний в аргоновой среде использовался сжатый газ по ГОСТ 10157-2016, подаваемый из газового баллона в герметичную камеру, с установленным в ней образцом.

\section{3. Экспериментальные подходы и методы анализа}

Для проведения экспериментов использовался стенд, конструкция которого подробно описана в статье [22].

В процессе экспериментов образцы нагревались при постоянных нагрузках, обеспечивающих номинальные растягивающие напряжения $\sigma=4.45,6.91$ и $9.36 \mathrm{MПа,} \mathrm{до} \mathrm{температур} \mathrm{673-1323} \mathrm{К.} \mathrm{Номинальное}$ напряжение рассчитывалось как отношение растягивающей нагрузки к начальному сечению образца. Поскольку предел текучести в этом температурном интервале для сплава Grade 5 больше 34 МПа, деформация образцов осуществлялась в режиме ползучести. Нагрев производился поэтапно. На каждом этапе в течении 10 с температура повышалась на $30 \mathrm{~K}$, после чего следовала 490 с выдержка. Согласно исследованиям Дорна [21], при таких условиях скорость ползучести определяется только изменением температуры.

Каждый эксперимент с нагруженными образцами дублировался идентичным экспериментом без нагрузки, что позволило определить удлинение образца за счет ползучести, исключив из общего удлинения вклад тепловой деформации образца и подвески.

Контроль удлинения образца осуществлялся только при равномерном удлинении рабочей части образца и прекращался в момент локализации деформации и образования шейки.

Результаты эксперимента были усреднены от трех тестов, проводимых при идентичных условиях.

Более подробно методика проведения эксперимента и обработки результатов описана в [20].

\section{4. Результаты и обсуждение}

Результаты экспериментов по определению скорости ползучести на установившейся стадии подвергнуты статистической обработке. Для аналитического описания результатов экспериментов использовали известную экспоненциальную зависимость [21]

$$
v=A \sigma^{n} \exp \left(-\frac{\Delta H}{R T}\right), 1 / \mathrm{c}
$$

где: $\Delta H-$ энергия активации установившейся ползучести, кДж/моль; $\sigma$ - растягивающее напряжение, МПа; $T$ - температура нагрева, K; $R$ - универсальная газовая постоянная, кДж/(моль $\cdot$ К); $A, n-$ эмпирические коэффициенты аппроксимации.

Для определения эмпирических коэффициентов $A$ и $n$ были использованы данные, полученные в экспе- 
риментах, проведенных при номинальных растягивающих напряжениях $\sigma=4.45$ и 9.36 МПа. Для повышения точности они определялись с помощью линеаризованной зависимости

$$
\ln v=\ln A+n \ln \sigma-\frac{\Delta H}{R T}
$$

Найденные коэффициенты обеспечивают точность описания экспериментальных данных с коэффициентом достоверности аппроксимации $\mathrm{R}^{2}>0.96$ в исследованных температурных интервалах.

Значения эмпирических коэффициентов, усредненные значения энергии активации и диапазоны температур, в которых они получены, приведены в Табл. 1.

На Рис. 1 в качестве примера показаны экспериментальные и расчетные данные скоростей ползучести при температурах установившегося режима ползучести для растягивающих напряжений $\sigma=9.36 \mathrm{MПа.}$

Статистическая обработка показала, что значения температуры нагрева $T_{0.2 / 1}$, при которых номинальные напряжения вызывают остаточную деформацию ползучести $0.2 \%$ в течение 1 часа выдержки, и значения условного предела кратковременной ползучести $\sigma_{0.2}$

Табл. 1. Значения энергии активации и эмпирических коэффициентов в формуле (1).

Table 1. The values of activation energy and the empirical coefficients in Eq. (1).

\begin{tabular}{|c|c|c|c|c|}
\hline $\begin{array}{c}\text { Среда } \\
\text { Environment }\end{array}$ & $\begin{array}{c}\Delta H, \text { кДж/моль } \\
\Delta H, \mathrm{~kJ} / \mathrm{mol}\end{array}$ & $A \cdot 10^{-4}$ & $n$ & $\begin{array}{c}\text { Диапазон } \\
\text { температур, } \mathrm{K} \\
\text { Теmperature } \\
\text { range, } \mathrm{K}\end{array}$ \\
\hline $\begin{array}{c}\text { Воздух } \\
\text { Air }\end{array}$ & 214 & 0.112 & 3.41 & $923-1323$ \\
\hline $\begin{array}{c}\text { Аргон } \\
\text { Argon }\end{array}$ & 320 & 11.91 & 3.37 & $1023-1323$ \\
\hline
\end{tabular}

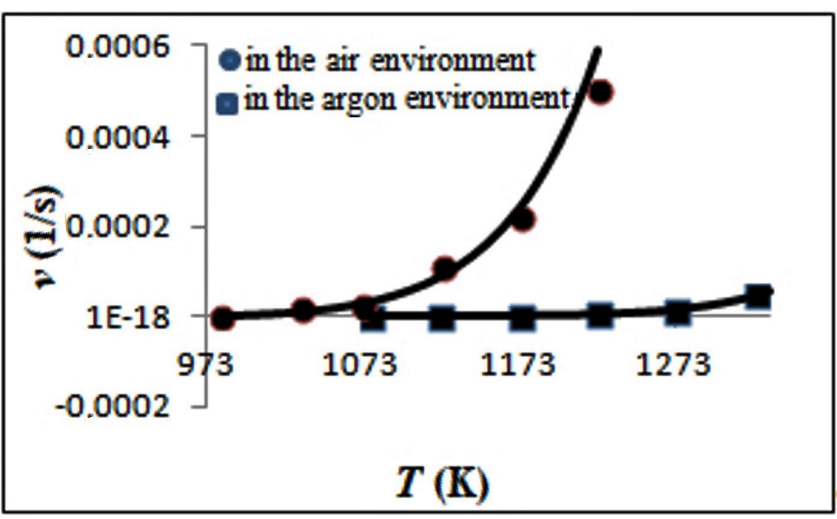

Рис. 1. Зависимость средней скорости ползучести от температуры нагрева при установившемся режиме ползучести и растягивающем напряжении $\sigma=9.36$ МПа. Точки на графиках обозначают усредненные экспериментальные данные, линии результаты расчета по формуле (1).

Fig. 1. The rate of steady-state creep as dependent on heating temperature at tensile stress $\sigma=9.36 \mathrm{MPa}$. The dots on the graphs denote averaged experimental data and the lines represent the results of calculation for Eq. (1). связаны полиноминальной зависимостью по формуле (3) с коэффициентом достоверности аппроксимации $\mathrm{R}^{2}>0.99$ :

$$
T_{0.2 / 1}=c_{1} \sigma_{0.2}^{2}+c_{2} \sigma_{0.2}+c_{3},
$$

где $c_{1}, c_{2}, c_{3}-$ коэффициенты аппроксимации, приведенные в Табл. 2.

Коэффициенты $c_{1}, c_{2}$ не имеют особого физического смысла, а коэффициент $c_{3}$ можно трактовать как температуру, при которой ползучесть начинает развиваться без действия внешних механических напряжений.

Экспериментальные данные по определению условного предела кратковременной ползучести $\sigma_{0.2}$ и рассчитанные по формуле (3) кривые приведены на Рис. 2.

Проведенные исследования выявили качественную аналогию влияния среды на процессы деформации ползучести для сплавов Grade 2, Ti-5AL и Grade 5 [20,24]. Для всех трех сплавов различия в значениях энергии активации были невелики (250-320 кДж/моль), что хорошо согласуется с результатами исследований [22,23]. Эксперименты показали, что сплав Grade 5, по сравнению с ранее исследованными сплавами, имеет более высокие температуры начала деформации ползучести (Рис. 3) и низкие скорости ползучести (Рис. 4).

Табл. 2. Значения эмпирических коэффициентов в формуле (2). Table 2. The values of the empirical coefficients in Eq. (2).

\begin{tabular}{|c|c|c|c|c|}
\hline $\begin{array}{c}\text { Среда } \\
\text { Environment }\end{array}$ & $c_{1}$ & $c_{2}$ & $c_{3}$ & $\begin{array}{c}\text { Диапазон } \\
\text { температур, K } \\
\text { Temperature range, K }\end{array}$ \\
\hline $\begin{array}{c}\text { Воздух } \\
\text { Air }\end{array}$ & -0.418 & -15.21 & 1103.5 & $923-1073$ \\
\hline $\begin{array}{c}\text { Аргон } \\
\text { Argon }\end{array}$ & -1.084 & -11.21 & 1210.5 & $1013-1173$ \\
\hline
\end{tabular}

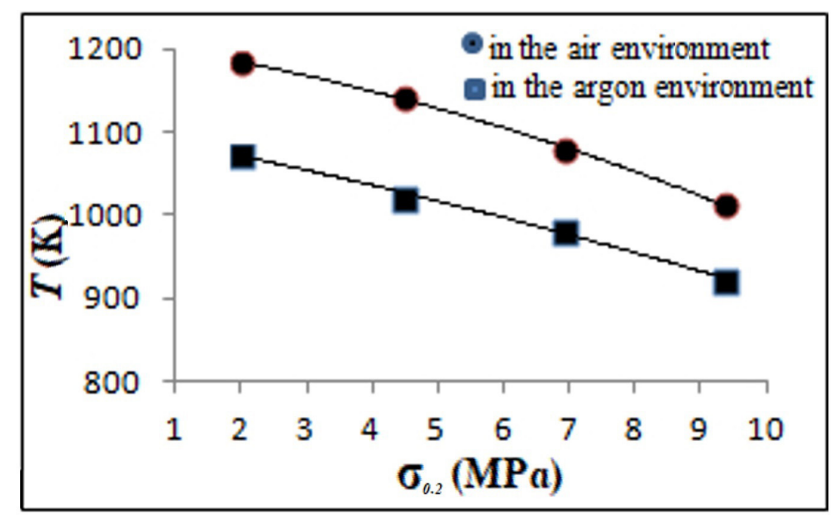

Рис. 2. Зависимости предела ползучести $\sigma_{0.2}$ от температуры нагрева для сплава Grade 5. Точки на графиках обозначают усредненные экспериментальные данные, линии - результаты расчета по формуле (2).

Fig. 2. Conventional short-term creep strength $\sigma_{0.2}$ as dependent on heating temperature for alloy Grade 5. The dots on the graphs denote averaged experimental data and the lines represent the results of calculation for Eq. (2). 


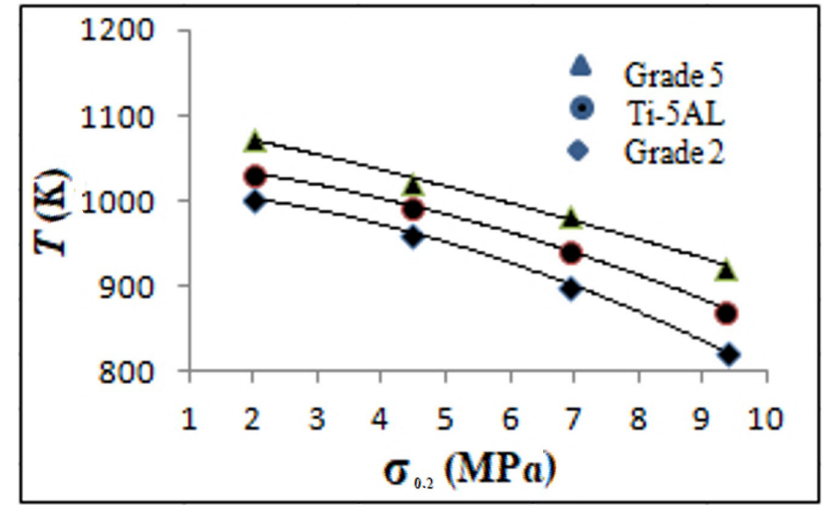

a

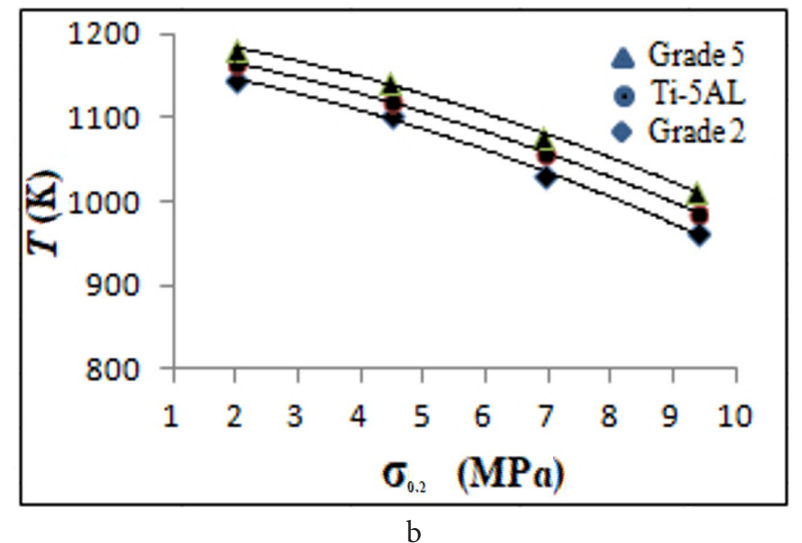

Рис. 3. Зависимости предела ползучести $\sigma_{0.2}$ от температуры нагрева для сплавов: в воздушной среде (a), в среде аргона (b). Точки на графиках обозначают усредненные экспериментальные данные, линии - результаты расчета по формуле (2).

Fig. 3. Conventional short-term creep strength $\sigma_{0.2}$ as dependent on heating temperature for alloys: in the air environment (a), in the argon environment (b). The dots on the graphs denote averaged experimental data and the lines represent the results of calculation for Eq. (2).

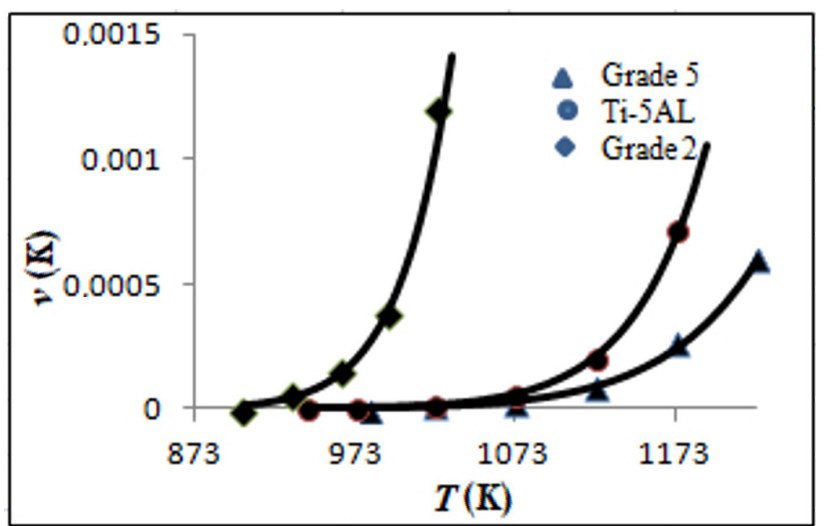

a

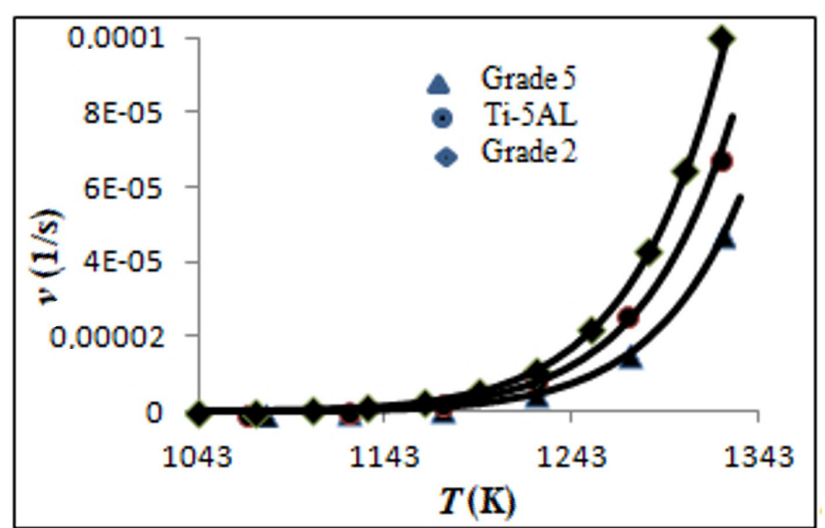

$\mathrm{b}$

Рис. 4.Зависимость средней скорости ползучести от температуры нагрева при установившемся режиме ползучести и растягивающем напряжении $\sigma=6.91$ МПа для сплавов: в воздушной среде (a), в среде аргона (b). Точки на графиках обозначают усредненные экспериментальные данные, линии результаты расчета по формуле (1).

Fig. 4. The rate of steady-state creep as dependent on heating temperature at tensile stress $\sigma=6.91 \mathrm{MPa}$ for alloys: in the air environment (a), in the argon environment (b). The dots on the graphs denote averaged experimental data and the lines represent the results of calculation for Eq. (1).

\section{5. Выводы}

В процессе проведенных исследований для сплава Grade 5 были определены значения эмпирических коэффициентов зависимости, скорости ползучести от температуры нагрева, напряжения растяжения и энергии активации ползучести, а также в зависимости определяющей начало деформации ползучести при исследованных температурах.

Установлено, что в сплаве Grade 5, по сравнению с ранее исследованными сплавами Grade 2 и Ti-5AL, деформация ползучести в обеих средах происходит при более высоких температурах и с более низкими скоростями.

\section{Литература/References}

1. J. J. Xiao, D. S. Li, X. Q. Li, P. Ding, K. Zhao, X.Z. Huang, M. J. Xu. Transactions of Nonferrous Metals Society of China. 25 (2), 420 (2015). $\underline{\text { Crossref }}$

2. D. L. Xiao, D. S. Li, C. Jin, T. Deng. Rare Metal Materials and Engineering. 42 (12), 2629 (2013).
3. J. Kumar, S. G. S. Raman, V. Kumar. Transactions of the Indian Institute of Metals. 69 (2), 349 (2016). Crossref

4. H. Yu, C. Dong, Z. Jiao, F. Kong, Y. Chen, Y. Su. Acta Metallurgica Sinica. 49 (11), 1311 (2013). $\underline{\text { Crossref }}$

5. D. McLean. Mechanical Properties of Metal. New York, London, John Wiley \& Sons (1962) 184 p.

6. F. Garofalo. Trans. AIME. 227, 351 (1963).

7. B. A. Kolachev. Met. Sci. Heat Treat. 4 (3-4), 93 (1999). Crossref

8. D. A. P. Reis, C. R. M. Silva, M. C. A. Nono, M. J. R. Barboza. Mater. Sci. Eng. A. 399 (1-2), 276 (2005). Crossref

9. A.M. Lokoshchenko, A.A. Il'In, A.M. Mamonov, V.V. Nazarov. Materials Science. 44 (5), 700 (2008). Crossref

10. G. P. Grabovetskaya, O. V. Zabudchenko, E. N. Stepanova. Russian Metallurgy (Metally). 3, 229 (2010). Crossref

11. M.C. Brandes, M. Baughman, M. J. Mills, J.C. Williams. Mater. Sci. Eng. A. 551, 13 (2012). Crossref

12. M. L. Wasz, F. R. Brotzen, R. B. McLellan, A. J. Griffin. Int. Mater. Rev. 41 (1), 1 (1996). Crossref

13. C. J. Beevers, M. R. Warren, D. V. Edmonds. Journal of the Less-Common Metals. 14 (4), 387 (1968). $\underline{\text { Crossref }}$ 
14. A.V. Makarov, E.S. Gorkunov, L. Kh. Kogan. Russian Journal of Nondestructive Testing, 43 (1), 21 (2007). Crossref

15. S. V. Smirnov, L. M. Zamaraev, P.P. Matafonov. Russian Metallurgy (Metally). 1, 67 (2010). Crossref

16. D. A. P. Reis, C. M. Neto, F. P. Neto, M. J. R. Barboza. SAE Technical Papers. 2007-01-2814 (2007). Crossref

17. D. A. P. Reis, C. M. Neto, M. D. C. A. Nono, M. J. R. Barboza, C. R. M. Da Silva, F. P. Neto. Proceedings of the 65th ABM International Congress, 18th IFHTSE Congress and 1st TMS/ABM International Materials Congress. 5, 4229 (2010).

18. S.V. Smirnov, L.M. Zamaraev, A.N. Zamyatin, P.P. Matafonov. Russian Metallurgy (Metally). 3, 255 (2012). Crossref

19. W. J. Evans, J.P. Jones, S. Williams. International Journal of Fatigue. 27, 1473 (2005). Crossref

20. S. V. Smirnov, L. M. Zamaraev. AIP Conf. Proc. 1915, 040069 (2017). Crossref

21. O. D. Sherby, J. L. Lytton, J. E. Dorn. Acta Metall. 5 (4), 219 (1957). Crossref

22. M. Doner, H. Conrad. Metall. Mater. Trans. A. 4 (12), 2809 (1973).

23. H. J. Frost, M. F. Ashby. Deformation-mechanism maps: the plasticity and creep of metals and ceramics. Oxford, New York, Sydney, Pergamon Press (1982) 166 p.

24. S. V. Smirnov, L.M. Zamaraev, P.P. Matafonov. International journal of applied and fundamental research. 11, 555 (2014). (in Russian) [С. В. Смирнов, Л.М. Замараев, П.П. Матафонов. Международный журнал прикладных и фундаментальных исследований. 11, 555 (2014).] 